\title{
Observation on ECG Changes in Thalassemia Major Patients
}

\author{
Dr. Dipti Neha ${ }^{1}$, Dr.Shashank Shekhar ${ }^{2}$, Dr.M.R.Akhouri ${ }^{3}$ \\ ${ }^{1}$ Junior Resident, ${ }^{2}$ Junior Resident, ${ }^{3}$ Associate Professor \\ Department of Paediatrics and Neonatology, Rajendra Institute of Medical Sciences (RIMS), Ranchi, Jharkhand
}

\begin{abstract}
:
Background: Cardiac complications are the most common cause of morbidity and mortality in thalassemia major patients which is due to multiple blood transfusions leading to cardiac haemosiderosis rather than the disease itself.

Objective: To evaluate ECG changes in thalassemia major patients for early detection of cardiac complications due to iron overload.

Methodology: This study was a hospital based prospective cross sectional study conducted from February 2015 to April 2016 in Department of Paediatrics and Neonatology, Rajendra Institute of Medical Sciences, Ranchi. In this study, 60 confirmed cases of thalassemia diagnosed on the basis of HPLC (High Performance Liquid Chromatography) were included for assessment of their cardiac profile and its correlation with serum ferritin level.

Results: Out of 60 thalassemic patients, one or more abnormal ECG findings were present in 31 children (51.6\%) and 29 children (48.3\%) had normal ECG. The most commonly observed ECG changes were sinus tachycardia, arrhythmias, left ventricular hypertrophy, ST- $T$ wave changes, conduction blocks and bradycardia.

Conclusion: Cardiac monitoring of thalassemia patients should be done at regular intervals. Due to its low cost, ECG can be utilised in areas where there is high prevalence of thalassemia for detecting cardiac complications especially when cardiac T2 MRI is not available.
\end{abstract}

Keywords: Electrocardiogram, Haemosiderosis

\section{Introduction}

Thalassemia are the most common genetic disorder worldwide. In India, over 40 million people carry genes for beta thalassemia. ${ }^{1}$ The thalassemias are heterogeneous group of disorders with genetically determined reduction in the rate of synthesis of one or more types of globin chain of haemoglobin (alpha or beta chain). ${ }^{2}$ In India, the diagnosis of thalassemia is often delayed due to lack of awareness regarding disease and the lack of facilities for diagnosis. The high mortality and morbidity in patients of thalassemia is the consequence of iron overload. Iron overload occurs mainly due to repeated blood transfusions and other factors like increased gastrointestinal absorption, ineffective erythropoiesis, lack of physiologic mechanism to excrete excess iron. Cardiac complications are the major cause of mortality in thalassemia patients. After about 10 years of transfusion, cardiac dysfunction secondary to haemosiderosis begins. ${ }^{3}$ Free iron damages cardiocytes inducing lipid peroxidation and lysosomal rupture. Eventually, most patients not receiving adequate iron chelation therapy develop cardiomyopathy, cardiac failure or arrhythmias secondary to myocardial haemosiderosis. ${ }^{4}$

\section{Methodology}

This study was done on 60 confirmed cases of thalassemia diagnosed on the basis of HPLC (High Performance Liquid Chromatography) of age group 8 months to 12 years attending inpatient and outpatient ward, Department of Paediatrics and Neonatology, Rajendra Institute of Medical Sciences, Ranchi., during the period of February 2015 to April 2016 for the assessment of their cardiac function.

The following information were taken from each patient: name, age, sex, religion, region, age at the time of diagnosis of thalassemia, total units of blood transfusions, whether the patient is on iron chelators, if yes, duration of therapy. Physical examination of the patient included general examination and cardiovascular system examination. Laboratory tests included pre and post transfusional haemoglobin, serum iron and ferritin level by chemiluminescence method and ECG. Patients having severe systemic illness and congenital heart disease were excluded from this study.

\section{Results}

In this study, out of 60 patients of thalassemia, majority of the patients (70\%) were males. Patients age ranged from 8 months to 12 years. Majority of patients were less than 5 years (56.6\%). Among 60 thalassemic children, the youngest case was diagnosed at 4 months of age and oldest at 18 months of age and mean age of 
diagnosis was around 7 months. Mean serum ferritin level in age group < 5 years who were not on iron chelators was $392.63 \mathrm{ng} / \mathrm{ml}$ and in those with iron chelators was $1815.46 \mathrm{ng} / \mathrm{ml}$. Out of 60 thalassemic patients, ECG changes were present in 31 children $(51.6 \%)$ and 29 children $(48.3 \%)$ had normal ECG.

Table 1: Case Distribution According To Ecg Changes Of The Patient

\begin{tabular}{|l|l|l|}
\hline ECG CHANGES & NO OF PATIENTS & $\%$ OF PATIENTS \\
\hline PRESENT & 31 & $51.6 \%$ \\
\hline ABSENT & 29 & $48.3 \%$ \\
\hline
\end{tabular}

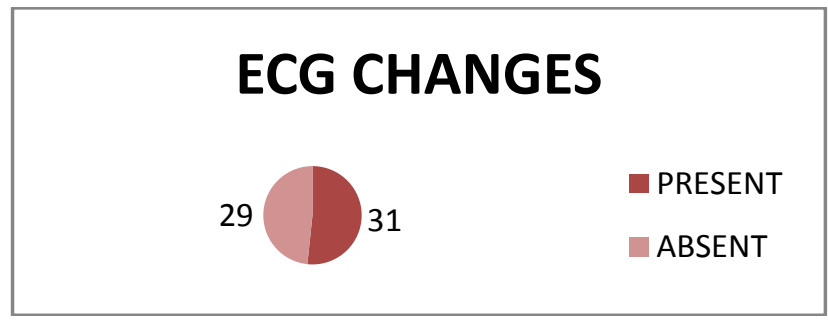

Fig 1: Ecg Changes

In patients with ECG changes , 24 children (40\%) had tachycardia, 3 patients (5\%) had bradycardia , 14 children (23.3\%) had arrhythmia, 12 patients (20\%) had Left Ventricular Hypertrophy (LVH) , 7 patients (11.6\%) with ST-T wave changes and 2 patients $(3.3 \%)$ had conduction block ( $1^{\text {st }}$ degree heart block).

Table 2 : Types Of Ecg Changes

\begin{tabular}{|l|l|l|}
\hline ECG CHANGES & NO OF PATIENTS & $\%$ OF PATIENTS \\
\hline TACHYCARDIA & 24 & $40 \%$ \\
\hline BRADYCARDIA & 3 & $5 \%$ \\
\hline ARRYTHMIA & 14 & $23.3 \%$ \\
\hline LVH & 12 & $20 \%$ \\
\hline ST-T WAVE CHANGES & 7 & $11.6 \%$ \\
\hline CONDUCTION BLOCKS & 2 & $3.3 \%$ \\
\hline
\end{tabular}

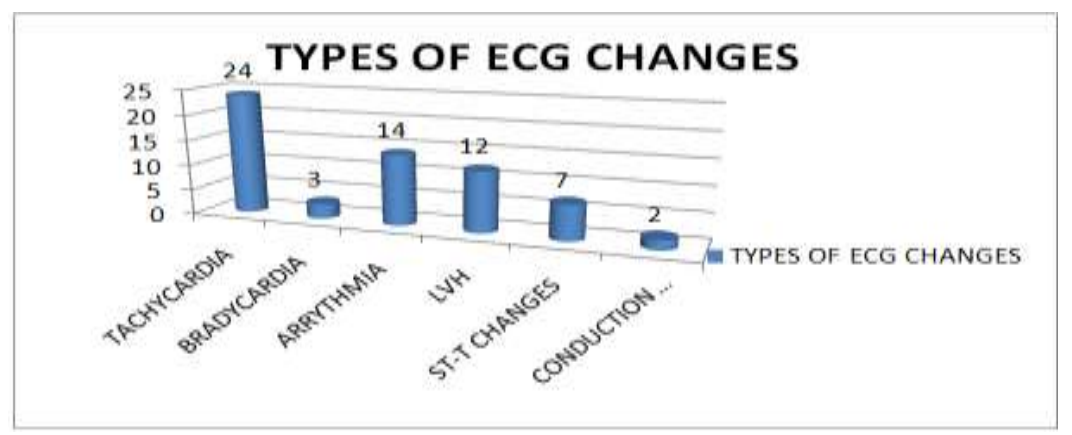

Table 3: Comparison Of Various Parameters In Patients According To Ecg Changes

\begin{tabular}{|l|l|l|l|}
\hline PARAMETERS & $\begin{array}{l}\text { PTS WITHOUT ECG CHANGES } \\
\text { (mean value) }\end{array}$ & $\begin{array}{l}\text { PTS WITH ECG } \\
\text { CHANGES (mean } \\
\text { value) }\end{array}$ & $\begin{array}{l}\text { P } \\
\text { value }\end{array}$ \\
\hline Mean Age $($ Years $)$ & 2.9 & 7.5 & $<0.05$ \\
\hline Total Units of BT & 25.5 & 75.4 & $<0.05$ \\
\hline S. Iron $(\mu \mathrm{g} / \mathrm{dl})$ & 144 & 277.7 & $>0.05$ \\
\hline S. Ferritin $(\mu \mathrm{g} / \mathrm{L})$ & 932.3 & 2957.3 & $<0.05$ \\
\hline
\end{tabular}




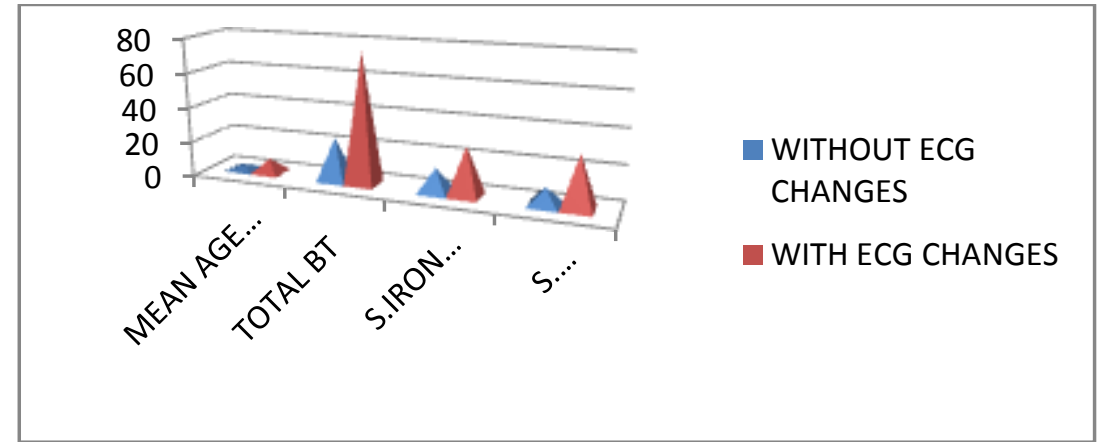

Comparative study between patients with normal ECG and with ECG changes present showed statistically significant difference $(\mathrm{p}<0.05)$ in relation to mean age, total units of BT, serum ferritin level which shows that ECG changes appear to be increasing with age, more units of BT, S. ferritin level. Though there was difference in the $\mathrm{S}$. iron level between the two groups but it was not statistically significant. $(\mathrm{p}>0.05)$.

\section{Discussion}

In the present study, minimum age of diagnosis was 4 months and maximum age as 18 months, the mean being around 7 months. This implies that thalassemia major patients present symptoms early in childhood, an early as 3 to 6 months with progressive pallor being the most common manifestation.

Table:4 Comparison of ECG changes among various studies

\begin{tabular}{|l|l|l|}
\hline Studies & Normal ECG (\%) & ECG changes present (\%) \\
\hline Ramazzotti A 2008 & $55 \%$ & $45 \%$ \\
\hline Mohammed SJ 2011 & $38.3 \%$ & $61.7 \%$ \\
\hline Present study & $48.3 \%$ & $51.6 \%$ \\
\hline
\end{tabular}

Table:5 Comparison of types of ECG changes in different studies

\begin{tabular}{|l|l|l|}
\hline ECG changes & Mohammed SJ 2011 & Present study \\
\hline Tachycardia & $52.2 \%$ & $40 \%$ \\
\hline Bradycardia & - & $5 \%$ \\
\hline Arrhythmia & - & $23.3 \%$ \\
\hline LVH & $29.7 \%$ & $20 \%$ \\
\hline ST-T wave changes & $22.5 \%$ & $11.6 \%$ \\
\hline Conduction blocks & $1 \%$ & $3.3 \%$ \\
\hline
\end{tabular}

Reza $1972^{7}$, Walter $1978^{8}$ reported the most common ECG changes in thalassemia being supraventricular tachycardia. Engel $1964^{9}$ reported that some of thalassemic patients reported pericarditis after the the age of 10 years.

Anna Ramazzatti et al $2008^{5}$ found most common ECG changes being T wave inversion(27\%), Right bundle branch block(RBBB) in $12 \%$, flat $\mathrm{T}$ wave in $7 \%$ of the patients.

RBBB.

Pepe a et al $2009^{10}$ reported the most common ECG changes being T- wave inversion, flat $\mathrm{T}$ waves and

In the present study, out of 60 thalassemics, 41 patients $(68.3 \%)$ were on chelation therapy, all were on single oral iron chelator, deferasirox and 19 patients (31.6\%) were not on chelation therapy. In the age group $<5$ years, iron overload in these patients as suggested by serum iron and ferritin level is less owing to less duration of illness and total number of blood transfusions. Serum iron and ferritin level in patients on iron chelators appears to be rising with age. This finding suggests that though the patients are on oral iron chelators, but serum iron and ferritin level is increasing due to increasing number of blood transfusions which contributes to iron overload in thalassemic patients and single oral iron chelators is not effective in reducing iron overload in these patients.

In the present study, comparison was made between the patients with ECG changes and those without ECG changes. It was found that there was statistically significant difference between the two groups in relation to mean age, total units of blood transfusions, serum ferritin level. There was definitive positive correlation with serum ferritin level showing ECG changes appears due to myocardial siderosis secondary to iron overload. However, there was difference related to serum iron in the two groups, but not statistically significant.

The above findings are suggestive of the fact that cardiac complications in thalassemia major increases with increasing total units of blood transfusions with increasing age. These blood transfusions combined with increased gastrointestinal absorption contributing to iron overload and myocardial iron deposition leading to cardiac dysfunction. 
Cardiac monitoring of the patients at regular intervals is required. ECG owing to its low cost can be used in areas where there is high prevalence of thalassemia and limited resources.

\section{Conclusion}

Thalassemia syndromes are important cause of morbidity and mortality in children. Though cardiac dysfunction secondary to iron overload in thalassemic patients may start early in life, clinical signs are not observed in most patients. Regular follow up for early detection and therefore timely treatment of such complications could improve the quality of life of these patients. There was strong association between high serum ferritin level and cardiac dysfunction. This stresses the need for intensive iron chelation therapy to be individually monitored to provide normal iron balance which should be sufficient to avoid cardiotoxicity and other associated complications due to iron overload.

\section{References}

[1]. Choudhary V.P, R. Kashyap, S.K. Acharya, N. Tandon, A. Saxena : Challenges in the management of Thalassemia, Mediquest Medicl Information series- 1997: Vol.15: No.3: 1-19

[2]. Sarnaik SA. Thalassemia and Related Haemoglobinopathies. Indian J paediatrics 1975; 12: 195-199

[3]. Telfer PT, Warburton F, Christou S, Hadjigavriel M, Sitarou M, Kolnagou A. (2009). Improved survival in thalassemia major patients on switching, from desferrioxamine to combined chelation therapy with desferrioxamine and deferiprone. Haematologica, Vol. 94(12), pp. 1777-8

[4]. Wood JC, Enriquez C, Ghugre N, Otto-Duessel M, Aguilar M, Nelson MD, Moats R, Coates TD Physiology and pathophysiology of iron cardiomyopathy in thalassemia. Ann N Y Acad Sci. 2005;1054:386-395.

[5]. Anna Ramzzooti, Aiessia, Pepe, Vencenza Pasitano, Barbara Scattini, Maria F.Santerelli, Luigi Landini, Daneil. March: Standarized $\mathrm{T} 2 *$ map of normal human heart to correct iron overload and fibrosis in thalassemia intermedia versus thalassemia major patients and electrocardiogram change in thalassemia major

[6]. Mohammed SJ, Al-Lami FH, Hussein MQ. Electrocardiographic changes among beta thalassemia major patients in ibn al-baladi thalassemia center-Baghdad. Al-Kindy Col Med J 2013; 9(1): 36-42

[7]. Reza Gharib, Sharman Ayazi: Electrocardiographic finding in Iranian children with severe chronic anemia. Clinic Pediatric 1972; 11:636.

[8]. Walter L. Henry, Arthur W. Nienhuis: Echocardiographic abnormalities in patients with transfusion dependant anemia and secondary myocardial iron deposition Am J Med 1978;

[9]. 64:547.

[10]. Engl MA, Erlandson M, Smith CA: Late cardiac complication of chronic severe refractory anemia with hemochromatosis. Circulation 1964; 30: 689

[11]. Pepe A, Positano V, Capra M, Maggio A, Myocardial scarring by delayed enhancement cardiovascular magnetic resonance in thalassaemia major.Heart. 2009;95:1688-1693 